\title{
Oligomycin induced the proteasomal degradation of cyclin D1 protein
}

\author{
Mai Kanai, Satoru Iba, Ryoko Okada, Etsu Tashiro and Masaya Imoto \\ We searched for compounds that affect the cyclin D1/retinoblastoma protein pathway from the in-house natural product library \\ using a recombinant adenovirus with the Cre/loxP-regulated cyclin D1 overexpression system, and we found that oligomycin \\ inhibited cell growth more effectively in cyclin D1-overexpressing SW480 cells than in control SW480 cells. We also found that \\ oligomycin reduced the expression levels of cyclin D1 protein and that this reduction is, at least in part, mediated by Thr-286 \\ phosphorylation-dependent proteasomal degradation.
}

The Journal of Antibiotics (2009) 62, 425-429; doi:10.1038/ja.2009.47; published online 3 July 2009

Keywords: cell growth inhibition; chemosensitivity; cyclin D1; pRB; E2F-1

\section{INTRODUCTION}

The transition from G1 to $S$ phase is regulated by cyclins, cyclindependent kinases $(\mathrm{CDK})$ and their inhibitor proteins. The protooncogenic function of cyclin D1 has been attributed in part to its role in promoting cell cycle progression. Cyclin D1 is a key cell cycle regulator of the $\mathrm{G} 1$ to $\mathrm{S}$ phase progression. The binding of cyclin D1 to cyclin-dependent kinase ( $\mathrm{cdk} 4$ or cdk6) leads to the phosphorylation of retinoblastoma protein $(\mathrm{pRB})$ subsequently triggering the release of E2F transcription factors to allow the transcription of genes required for the G1 to $S$ phase progression of the cell cycle. In several types of human cancer, there is abundant evidence that disturbances of specific cyclins, CDKs or CDK inhibitory proteins enhance tumor cell growth. ${ }^{1-3}$ In particular, disruption of the regulation of the cyclin D1/pRB/E2F pathway, overexpression of cylin D1, loss of pRB function and amplification of E2F-1 are often observed in several types of cancer. ${ }^{2,4,5}$ Although recent reports have shown that some antitumor drugs may affect the cyclin D1/pRB/E2F pathway, ${ }^{6-8}$ it is unclear which types of antitumor drugs induced the disruption of this pathway. In this study, we searched for compounds that inhibit cell growth selectively in cyclin D1-overexpressing human colorectal carcinoma cell lines (SW480 and LoVo) using a recombinant adenovirus with the Cre/loxP-regulated expression system.

\section{MATERIALS AND METHODS}

\section{Cell culture and reagents}

Human embryonic kidney HEK293 cells were grown in Dulbecco's Modified Eagle's Medium supplemented with $10 \%$ fetal bovine serum (FBS). Human colon cancer SW480 and LoVo cell lines were maintained in RPMI 1640 medium with 5\% FBS. Adriamycin, camptothecin, etoposide, tunicamycin, wortmannin and bafilomycin Al were purchased from Sigma Chemical (St Louis, MO, USA). Vinblastine and taxol were obtained from Wako Pure
Chemical Industries, Ltd (Tokyo, Japan). Thapsigargin was obtained from Santa Cruz Biotechnology (Santa Cruz, CA, USA). Oligomycin was obtained from Calbiochem (San Diego, CA, USA). Leptomycin B was kindly supplied by Dr Minoru Yoshida (RIKEN, Japan). Inostamycin was isolated from the fermentation broth of Streptomyces. ${ }^{9}$

Adenoviral vectors and adenoviral vector-mediated expression To exogenously express cyclin D1, we used adenovirus Cre/loxP-regulated expression vectors (TaKaRaBio, Shiga, Japan). In these vectors, pAxCALNLw, a stuffer DNA fragment sandwiched by two loxP sequences, is located just upstream of cyclin D1 cDNA and interferes with the expression of cyclin D1. Recombinant adenoviruses (adenovirus expressing wild-type cyclin D1 (Ad-cyclin D1) and adenovirus expressing Cre-recombinase (Ad-Cre)) were grown and concentrated in HEK293 cells. Infection was carried out by the addition of recombinant adenoviruses to serum-containing media following the manufacturer's instructions. SW480 and LoVo cells seeded on 6- or 96-well plates were incubated with virus-containing media at the indicated multiplicity of infection (MOI) at $37^{\circ} \mathrm{C}$ for $1 \mathrm{~h}$. After $1 \mathrm{~h}$ of incubation at $37^{\circ} \mathrm{C}$, the $1.5 \mathrm{ml}$ (6-well plates) or $100 \mu$ l (96-well plates) of the fresh medium containing $5 \%$ FBS were added.

\section{Western blotting}

Cells were lysed in lysis buffer (50 mм HEPES ( $\mathrm{pH} 7.2$ ), $150 \mathrm{~mm} \mathrm{NaCl}, 2.5 \mathrm{~mm}$ EGTA, $1 \mathrm{~mm}$ EDTA, $1 \mathrm{~mm}$ DTT, 0.1\% Tween 20, $10 \mathrm{~mm} \beta$-glycerophosphate, $1 \mathrm{~mm} \mathrm{NaF}, 0.1 \mathrm{~mm} \mathrm{Na} \mathrm{Vo}_{4}, 10 \%$ glycerol and $0.1 \mathrm{~mm}$ PMSF) for $30 \mathrm{~min}$ on ice and centrifuged at 15000 r.p.m. for $15 \mathrm{~min}$ to yield the soluble cell lysates. For immunoblotting, $50 \mu \mathrm{g}$ proteins of cell lysates were subjected to $10 \%$ SDSpolyacrylamide gel electrophoresis. Proteins were transferred onto a polyvinylidene fluoride membrane and then incubated with appropriate antibodies for $1 \mathrm{~h}$. Enhanced chemiluminescence (Millipore, Billerica, MA, USA) was used to visualize the immunoblot signals. 
a

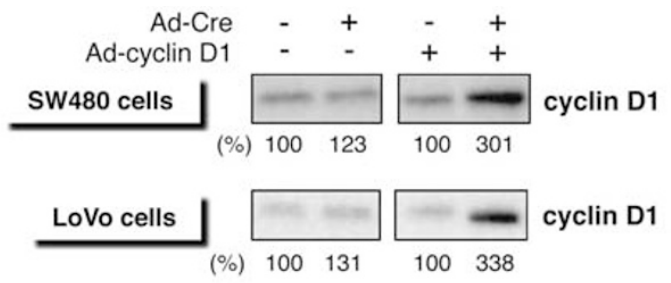

C
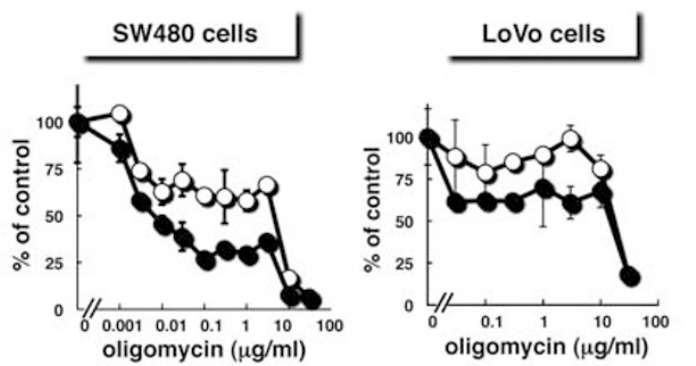

Cre (+)
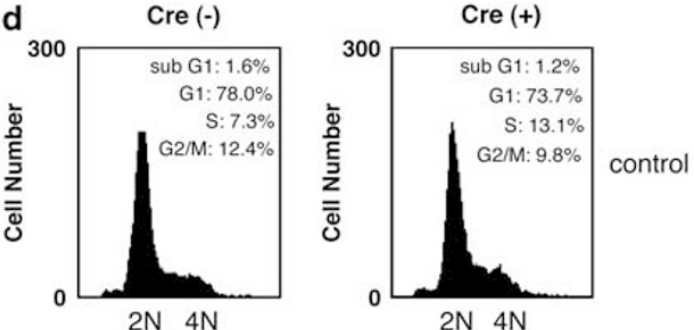

$2 \mathrm{~N} 4 \mathrm{~N}$

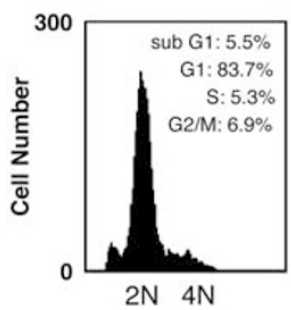

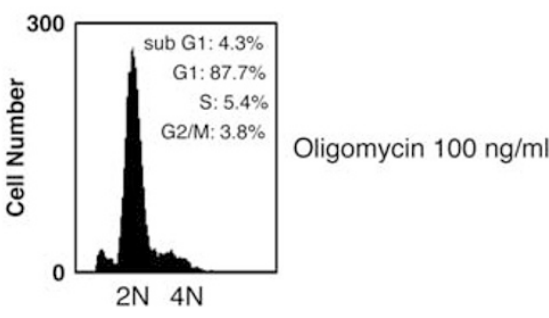

b
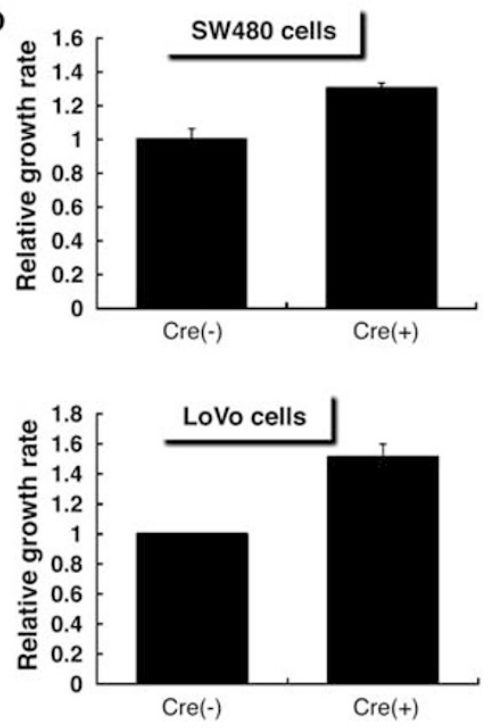

Figure 1 Effect of oligomycin on the growth of cyclin D1-overexpressing cells and control cells. SW480 cells and LoVo cells were infected with Ad-cyclin D1 ( $\mathrm{MOI} 3$ ) together with or without Ad-Cre (MOI 0.5). (a) At $48 \mathrm{~h}$ after infection, the protein expression of cyclin D1 was detected by western blotting using anti-cyclin D1 antibody (Santa Cruz Biotechnology). The bands of cyclin D1 were quantified by densitometry, and the results were normalized to control, which was arbitrarily set to 1.0 (below the panel). (b) At $48 \mathrm{~h}$ after infection, cell growth was measured by the MTT assay. Values represent the means $\pm \mathrm{s}$.d. (bars) obtained from triplicate determinations. (c) At $48 \mathrm{~h}$ after infection, cells were treated with oligomycin, and cells were further incubated for $48 \mathrm{~h}$. Cell growth was measured by the MTT assay (Ad-cyclin D1 alone (Cre(-)); open circle, Ad-cyclin D1/Ad-Cre (Cre(+)); closed circle). Values represent the means \pm s.d. (bars) obtained from triplicate determinations. (d) SW480 cells were infected with Ad-cyclin D1 (MOI 3) together with or without Ad-Cre (MOI 0.5). At $48 \mathrm{~h}$ after infection, cells were treated with $100 \mathrm{ng} \mathrm{ml}^{-1}$ of oligomycin and further incubated for $48 \mathrm{~h}$, and the DNA contents were then analyzed by flow cytometer. (e) SW480 cells were infected with Ad-cyclin D1 (MOI 3) together with or without Ad-Cre (MOI 0.5). At 48h after infection, cells were treated with the indicated concentration of oligomycin and further incubated for $48 \mathrm{~h}$, and then the cell viability was determined by trypan blue dye exclusion assay (Ad-cyclin D1 alone (Cre(-)); open circle, Ad-cyclin D1/Ad-Cre (Cre(+)); closed circle).

Figure 2 Oligomycin induced the degradation of cyclin D1 protein. (a) SW480 cells were infected with Ad-cyclin D1 (MOI 3) together with or without Ad-Cre ( $\mathrm{MOI}$ 0.5). At $48 \mathrm{~h}$ after infection, cells were treated with oligomycin at the indicated doses. At $48 \mathrm{~h}$ after treatment, cells were lysed and the expression levels of cell cycle progression-related proteins were detected by western blotting using anti-cyclin D1, anti-E2F-1 and anti-CDK2 (Santa Cruz Biotechnology), anti-pRB (BD Biosciences, Franklin Lakes, NJ, USA) and anti-cyclin E (Upstate, Charlottesville, VA, USA) antibodies. (b) SW480 cells were infected with Ad-cyclin D1 ( $\mathrm{MOI} 3$ ) together with Ad-Cre ( $\mathrm{MOI} 0.5$ ). At $48 \mathrm{~h}$ after infection, cells were treated with indicated concentrations of oligomycin for a further 24 and $48 \mathrm{~h}$, and then cyclin D1 mRNA was analyzed by real-time quantitative RT-PCR. GAPDH mRNA was measured for internal control and relative Cyclin D1 mRNA was calculated as Cyclin D1/GAPDH. The data are expressed as means \pm s.d. of three independent experiments. (c) SW480 cells were infected with Ad-cyclin D1 ( $\mathrm{MOI} 3$ ) together with Ad-Cre ( $\mathrm{MOI} 0.5)$. At $48 \mathrm{~h}$ after infection, cells were treated with oligomycin in the presence or absence of MG132. At 48 h after treatment, cells were lysed and the expression levels of cyclin D1 protein were detected by western blotting using an anticyclin D1 antibody. Percentages represent the relative expression level of cyclin D1 compared with treatment with $\mathrm{MeOH}$ and normalization to tubulin. 
Table 1 Effect of natural anti-tumor drugs on the growth of cyclin D1overexpressing SW480 cells (Cre(+)) and control SW480 cells (Cre(-))

\begin{tabular}{lccc}
\hline & \multicolumn{2}{c}{$C_{50}\left(\mathrm{mg} \mathrm{m}^{-1}\right)$} & \\
\cline { 2 - 3 } Antitumor drug & Cre (-) & Cre (+) & Cre (-)/Cre (+) \\
\hline Adriamycin & 0.75 & 0.64 & 1.17 \\
Camptothecin & 0.78 & 0.89 & 0.88 \\
Etoposide & 47 & 20 & 2.35 \\
Inostamycin & 0.49 & 0.48 & 1.02 \\
Thapsigargin & 0.07 & 0.05 & 1.40 \\
Tunicamycin & 0.45 & 0.36 & 1.25 \\
Leptomycin B & 0.36 & 0.56 & 0.64 \\
Vinblastine & $>3$ & $>3$ & - \\
Taxol & $>10$ & $>10$ & 0.78 \\
Wortmannin & 25.6 & 32.8 & 2.11 \\
Bafilomycin A1 & 2.17 & 1.03 & 758.57 \\
Oligomycin & 5.31 & 0.007 & \\
\hline
\end{tabular}

Abbreviation: MOI, multiplicity of infection.

SW480 cells were infected with Ad-cyclin D1 (MOI 3) together with or without Ad-Cre (MOI 0.5). Values are the means of three samples.

\section{MTT cell growth assay}

The cells were seeded at $3 \times 10^{3}$ cells (SW480 cells) or $1.5 \times 10^{3}$ cells (LoVo cells) in $100 \mu \mathrm{l}$ of growth medium in 96 -well plates. The following day, the cells were infected with Ad-Cyclin D1 with or without Ad-Cre. At $48 \mathrm{~h}$ after infection, drugs were added and the cells were further incubated for $48 \mathrm{~h}$. A total of $10 \mu \mathrm{l}$ of $5 \mathrm{mg} \mathrm{ml}^{-1}$ 3-(4,5-dimethylthiazol-2-yl)-2,5-diphenyltetrazolium bromide (MTT) in phosphate-buffered saline was added to each well, and the plate was incubated at $37^{\circ} \mathrm{C}$ for $4 \mathrm{~h}$. Then, $100 \mu \mathrm{l}$ of DMSO was added to the cells. The amount of Formazan formed was measured at $570 \mathrm{~nm}$ using a MRP-A4 microplate reader (Tosho, Tokyo, Japan).

\section{Real-time RT-PCR}

Total RNA $(2 \mu \mathrm{g})$ isolated from cells with TRIzol reagent (Invitrogen, Carlsbad, CA, USA) was reverse transcribed with $2 \mu$ g oligo(dT) primer using MMLVRT/HT point mutant reverse transcriptase (Promega, Madison, MA, USA) at $42^{\circ} \mathrm{C}$ for $1 \mathrm{~h}$. Real-time reverse transcriptase (RT)-PCR assay was performed using SYBR Premix Ex Taq (TaKaRaBio), and PCR amplification was carried out in a MJ mini personal thermal cycler (Bio-Rad, San Diego, CA, USA). Relative mRNA levels of Cyclin D1 were calculated by MJ opticon monitor analysis version 3.1 (Bio-Rad).

\section{Plasmid construction and transfection}

Flag-Cyclin D1 cDNA was generated from pcDNA3-Cyclin D1 by PCR using the synthetic primers $5^{\prime}$-GTGTCCTCGACCACAAGGTAGAACAGCAGCAG

a
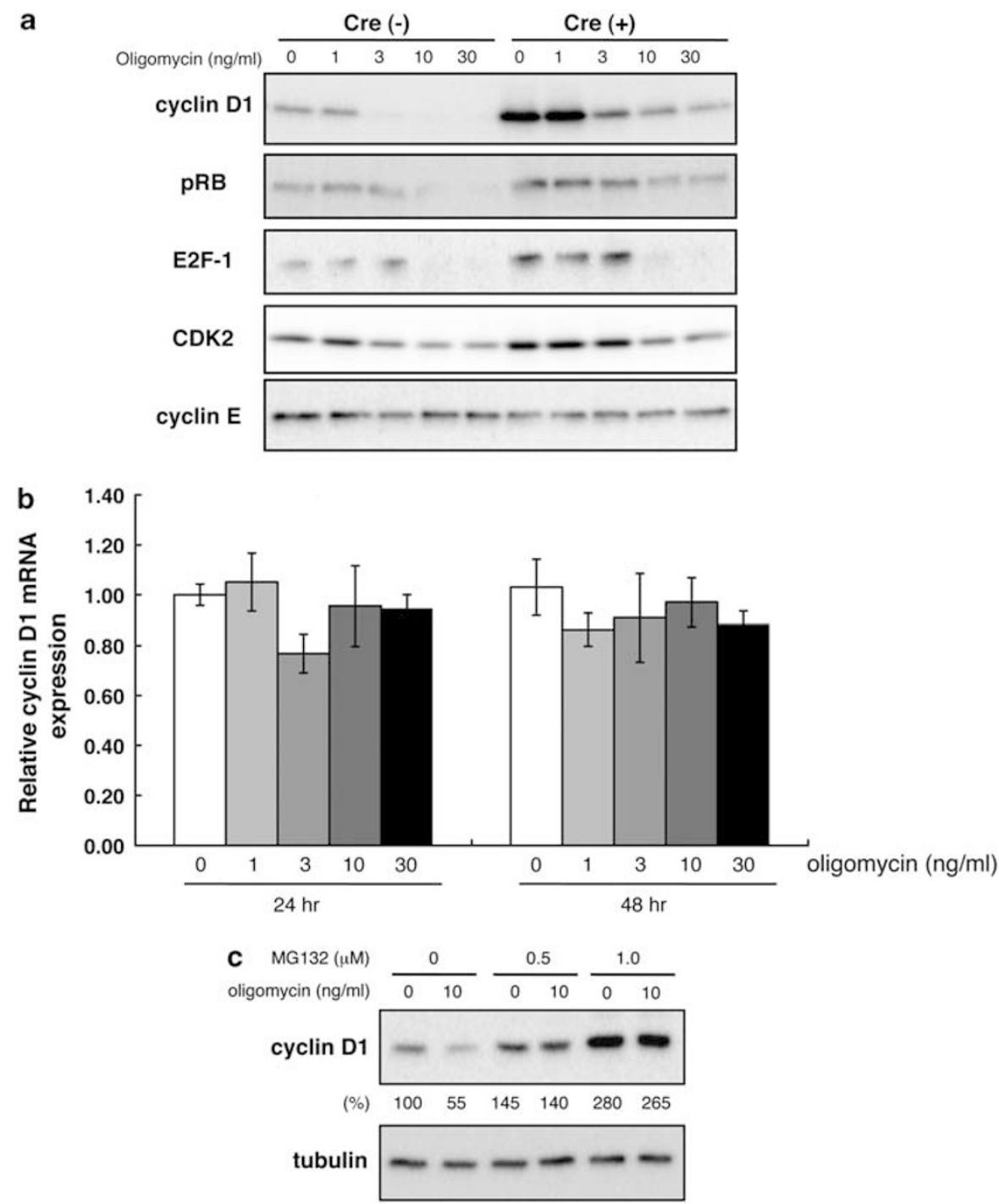


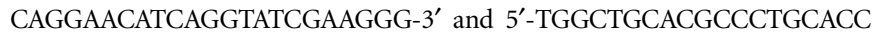
TGTAGACTTTCGAAGGG- ${ }^{\prime}$. The amplified PCR product was inserted into the pBlueScript vector to construct pBS-Flag-Cyclin D1. Point mutant T286A of Flag-Cyclin D1 was generated by PCR using sense primer 5'-CGTGCAGC CACCCGCGCGTTCGGTCCGGT- $3^{\prime}$ and antisense primer $5^{\prime}$-ACCTGGACC GAACGCGCGGGTGGCTGCACG-3'. Both pBS-Flag-Cyclin D1 and pBS-FlagCyclin D1 T286A were digested with HindIII and inserted into the pcDNA3 vector. For transient transfection, HEK293 T cells were seeded at $2.5 \times 10^{5}$ cells per well in 12-well plates. The following day, HEK293T cells were transfected with $0.3 \mu \mathrm{g}$ of plasmid using lipofectamine 2000 (Invitrogen) in OPTIMEM according to the manufacturer's instructions.

\section{Flow cytometry}

The cells were collected by trypsinization, combined with cells floating in the medium, and then stained with propidium iodide. The cell suspension was analyzed using an EPICS Elite and EXPO32 software analysis program (Beckman Coulter, Fullerton, CA, USA).

\section{RESULTS AND DISCUSSION}

To overexpress cyclin D1 protein in human colon cancer cell lines SW480 and LoVo, we used the recombinant adenovirus with the Cre/loxP-regulated expression system. The cells were infected with a combination of Ad-cyclin D1/Ad-Cre $(\mathrm{Cre}(+))$ or Ad-cyclin D1 alone $(\mathrm{Cre}(-))$, and their cyclin D1 expression levels were evaluated by western blot analysis. We found that expression levels of cyclin D1 in SW480 and LoVo cells were increased $48 \mathrm{~h}$ after infection with a combination of recombinant adenovirus cyclin D1 (Ad-cyclin D1; MOI 3) and Cre-recombinase (Ad-Cre; MOI 0.5) (Figure 1a), and a high level of cyclin D1 expression was maintained for $96 \mathrm{~h}$ after adenovirus infection, as judged from western blotting experiments. In addition, the growth rate of Ad-cyclin D1/Ad-Cre $(\mathrm{Cre}(+))$ cells was approximately 1.5-fold higher than that of Ad-cyclin D1 alone $($ Cre $(-))$ cells (Figure 1b). Using this system, we next examined the effects of twelve known natural antitumor drugs-adriamycin (topoisomerase II inhibitor), camptothecin (topoisomerase I inhibitor), etoposide (topoisomerase II inhibitor), inostamycin (phosphatidylinositol turnover inhibitor), thapsigargin $\left(\mathrm{Ca}^{2+}\right.$-ATPase inhibitor), tunicamycin ( $N$-linked glycosylation inhibitor), leptomycin B (CRM1 inhibitor), vinblastine (microtubule inhibitor), taxol (tubulin inhibitor), wortmannin (PI3 kinase inhibitor), bafilomycin A1 (V-ATPase inhibitor) and oligomycin $\left(\mathrm{F}_{0} \mathrm{~F}_{1}\right.$-ATPase inhibitor) - on the growth of cyclin D1-overexpressing cells and control cells by the MTT cell growth assay. We found that oligomycin inhibited cell growth more effectively in cyclin D1-overexpressing SW480 cells $(\mathrm{Cre}(+))$ than in control SW480 cells $(\mathrm{Cre}(-)$ ) (Table 1 and Figure 1c). The $\mathrm{IC}_{50}$ value of oligomycin for cell growth inhibition in cyclin D1-overexpressing SW480 cells (Ad-cyclin D1; MOI 3, Ad-Cre; MOI 0.5) was approximately 750 -fold lower than in control SW480 cells (Ad-cyclin D1; MOI 3). Similar results were observed in cyclin D1-overexpressing LoVo cells and control LoVo cells (Figure 1c).

The growth inhibition of cyclin D1-overexpressing SW480 cells caused by oligomycin (up to $1 \mu \mathrm{g} \mathrm{ml}^{-1}$ ) did not relate to the cytotoxic effect of the drug, as determined by trypan blue dye exclusion assay and flow cytometric analysis (Figures 1d and e). Next, we examined the effect of oligomycin on the expression levels of cell cycle-related proteins in cyclin D1-overexpressing cells $(\mathrm{Cre}(+))$ and control cells $(\mathrm{Cre}(-))$. As shown in Figure 2a, oligomycin reduced the expression levels of cyclin D1 protein in both cyclin D1-overexpressing SW480 cells and control cells. The expressions of pRB, E2F-1 and CDK2 were also decreased by oligomycin with a similar profile in both cells, whereas the expression profiles of cyclin E were not affected by
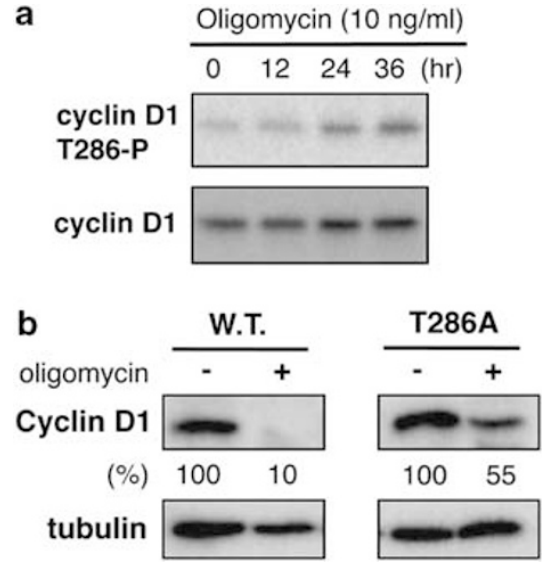

Figure 3 Involvement of T286 phosphorylation in oligomycin-induced decrease in cyclin D1 expression. (a) SW480 cells were treated with $10 \mathrm{ng} \mathrm{ml}^{-1}$ oligomycin. At the indicated times after treatment, cells were lysed and T286-phosphorylated cyclin D1 was detected by western blotting using anti-phospho-cyclin D1 (Thr-286) antibody (Cell Signaling Technology, Danvers, MA, USA). (b) HEK293T cells were transfected with Flag-tagged cyclin D1 wild-type (Flag-cyclin D1 WT) or Flag-tagged T286A mutant of cyclin D1 (Flag-cyclin D1 T286A) for $24 \mathrm{~h}$, and they were also incubated with or without oligomycin $\left(10 \mathrm{ng} \mathrm{ml}^{-1}\right)$ for $48 \mathrm{~h}$. Protein samples were detected by western blotting using an anti-Flag antibody (M2; Sigma-Aldrich Corp., St Louis, MO, USA). Percentages represent the relative expression level of Flag-cyclin D1 compared with treatment with $\mathrm{MeOH}$ and normalization to tubulin.

oligomycin. The growth rate of cyclin D1-overexpressing cells was higher than that of control cells (Figure 1b), indicating that the growth properties of cyclin D1-overexpressing cells might be more sensitive to the expression levels of cyclin D1 than that of control cells, which may explain the difference in the growth inhibition rate between cyclin D1overexpressing cells and control cells.

Gemin et al. also reported that treatment of HL60 cells with oligomycin resulted in a reduction in the amount of endogenous cyclin D1 protein; ${ }^{10,11}$ however, it has not yet been reported how oligomycin reduced the expression levels of cyclin D1. We found that the mRNA level of cyclin D1 was not affected by treatment with oligomycin when the cyclin D1 protein level was decreased (Figure 2b), indicating that decreased expression of cyclin D1 protein did not occur at the transcriptional level. On the other hand, oligomycin failed to reduce the expression level of cyclin D1 in cyclin D1-overexpressing SW480 cells when cells were treated with MG132, an inhibitor of proteasome (Figure 2c). These results indicated that the oligomycin-induced decrease in cyclin D1 expression levels was because of the proteasomal degradation of cyclin D1.

It is known that cyclin D1 is ubiquitinated and degraded through its phosphorylation at Thr-286 by glycogen synthase kinase-3 $\beta$ $($ GSK3 $\beta),{ }^{12}$ IKB kinase $\alpha$ (IKK $\left.\alpha\right),{ }^{13} \mathrm{p} 38^{14}$ or extracellular signalregulated kinase 1/2 (ERK1/2). ${ }^{15}$ As shown in Figure 3, oligomycin induced phosphorylation at Thr-286 in cyclin D1 (Figure 3a), and elimination of this site through point mutation (Ala for Thr-286 substitution: T286A) markedly but did not completely stabilize cyclin D1 (Figure 3b); therefore, although at present we do not know which kinase is responsible for phosphorylation at Thr-286 in cyclin D1 after oligomycin treatment, oligomycin-induced reduction of cyclin D1 expression is, at least in part, mediated by Thr-286 phosphorylation-dependent proteasomal degradation. Other degradation motifs of cyclin D1, such as the Arg-X-X-Leu destruction box, ${ }^{16}$ may also be involved in oligomycin-induced degradation of cyclin D1 protein. 
Apoptolidin as well as oligomycin is also reported to be an inhibitor of the $\mathrm{F}_{0} \mathrm{~F}_{1}$-ATPase inhibitor, and is identified on the basis of its ability to selectively kill E1A- and E1A/E1B19K-transformed rat glial cells while not killing untransformed glial cells. ${ }^{17} \mathrm{E} 1 \mathrm{~A}$ alone is sufficient to activate the cell cycle, inducing the expression of genes required for DNA synthesis and repeated rounds of cell division. Binding of $\mathrm{pRB}$ by E1A is intimately linked with these activities. E1B products alone show no ability to induce proliferation; however, the proliferation of cells in response to E1A is often quite limited in the absence of E1B expression; ${ }^{18}$ therefore, apoptolidin is also related to the cyclin D1/ $\mathrm{pRB} / \mathrm{E} 2 \mathrm{~F}$ pathway. Although further studies on the biological activities of oligomycin are required, the $\mathrm{F}_{0} \mathrm{~F}_{1}$-ATPase inhibitors, such as oligomycin and apoptolidin, affect the disruption of the cyclin D1/ $\mathrm{pRB} / \mathrm{E} 2 \mathrm{~F}$ pathway.

\section{ACKNOWLEDGEMENTS}

This study was supported in part by the Global COE program for Human Metabolomic Systems Biology from MEXT, Japan.

1 Draetta, G. F. Mammalian G1 cyclins. Curr. Opin. Cell Biol. 6, 842-846 (1994).

2 Hunter, T. \& Pines, J. Cyclins and cancer. II: cyclin D and CDK inhibitors come of age. Cell 79, 573-582 (1994).

3 Sherr, C. J. G1 phase progression: cycling on cue. Cell 79, 551-555 (1994).

4 Nevins, J. R. The Rb/E2F pathway and cancer. Hum. Mol. Genet. 10, 699-703 (2001).
5 Saito, M. et al. Amplification of the E2F1 transcription factor gene in the HEL erythroleukemia cell line. Genomics 25, 130-138 (1995).

6 Endo, N. et al. Herbimycin A induces G1 arrest through accumulation of p27(Kip1) in cyclin D1-overexpressing fibroblasts. Biochem. Biophys. Res. Commun. 267, 54-58 (2000).

7 Pirkmaier, A., Yuen, K., Hendley, J., O'Connell, M. J. \& Germain, D. Cyclin d1 overexpression sensitizes breast cancer cells to fenretinide. Clin. Cancer Res. 9 , 1877-1884 (2003).

8 Dong, Y. B., Yang, H. L., Elliott, M. J. \& McMasters, K. M. Adenovirus-mediated E2F-1 gene transfer sensitizes melanoma cells to apoptosis induced by topoisomerase II inhibitors. Cancer Res. 62, 1776-1783 (2002).

9 Imoto, M. et al. Isolation and structure determination of inostamycin, a novel inhibitor of phosphatidylinositol turnover. J. Nat. Prod. 53, 825-829 (1990).

10 Sweet, S. \& Singh, G. Accumulation of human promyelocytic leukemic (HL-60) cells at two energetic cell cycle checkpoints. Cancer Res. 55, 5164-5167 (1995).

11 Gemin, A., Sweet, S., Preston, T. J. \& Singh, G. Regulation of the cell cycle in response to inhibition of mitochondrial generated energy. Biochem. Biophys. Res. Commun. 332, 1122-1132 (2005)

12 Diehl, J. A., Cheng, M., Roussel, M. F. \& Sherr, C. J. Glycogen synthase kinase-3beta regulates cyclin D1 proteolysis and subcellular localization. Genes Dev. 12, 34993511 (1998).

13 Kwak, Y. T. et al. IkappaB kinase alpha regulates subcellular distribution and turnover of cyclin D1 by phosphorylation. J. Biol. Chem. 280, 33945-33952 (2005).

14 Casanovas, 0 . et al. Osmotic stress regulates the stability of cyclin D1 in a p38SAPK2dependent manner. J. Biol. Chem. 275, 35091-35097 (2000).

15 Okabe, $\mathrm{H}$. et al. A critical role for FBXW8 and MAPK in cyclin D1 degradation and cancer cell proliferation. PLOS ONE 1, e128 (2006).

16 Agami, R. \& Bernards, R. Distinct initiation and maintenance mechanisms cooperate to induce G1 cell cycle arrest in response to DNA damage. Cell 102, 55-66 (2000).

17 Kim, J. W., Adachi, H., Shin-ya, K., Hayakawa, Y. \& Seto, H. Apoptolidin, a new apoptosis inducer in transformed cells from Nocardiopsis sp. J. Antibiot. (Tokyo) 50 628-630 (1997).

18 Moran, E. Interaction of adenoviral proteins with pRB and p53. FASEB J. 7, 880-885 (1993). 